\title{
Stochastic Resonance in Perspective
}

\author{
M.I.Dykman \\ Department of Physics, Michigan State University, East Lansing, Michigan 48824-1116, \\ D.G.Luchinsky \\ All-Union Research Institute for Metrological Service, 117965 Moscow, Russia, \\ R.Mannella \\ Dipartimento di Fisica, Università di Pisa, Piazza Torricelli 2, 56100 Pisa, Italy \\ P.V.E.McClintock, N.D.Stein, and N.G.Stocks ${ }^{1}$ \\ School of Physics and Materials, Lancaster University, \\ Lancaster LA1 $4 Y B$, United Kingdom.
}

\begin{abstract}
We outline the historical development of stochastic resonance (SR), a phenomenon in which the signal and/or the signal-to-noise ratio in a nonlinear system increase with increasing intensity of noise. We discuss basic theoretical ideas explaining and describing SR, and we review some revealing experimental data that place SR within the wider context of statistical physics. We emphasize the close relationship of SR to some effects that are well known in condensed-matter physics.
\end{abstract}

\footnotetext{
${ }^{1}$ Now at: Department of Engineering, University of Warwick, Coventry, CV4 7AL, United Kingdom.
} 


\section{Introduction}

The term stochastic resonance (SR) is used to describe a group of phenomena in which the fluctuations of a nonlinear system enhance its response to a regular (e.g. periodic) force. The response at first increases with increasing noise intensity and then decreases again, and the same is often true of the signal-to-noise ratio as well. Over the last decade SR has attracted considerable attention from the scientific community, and about a hundred papers on the issue have been published. The subject has been discussed at many meetings, including a topical international conference whose proceedings [1] describe the state of the art as at mid-1992. Since that time, several interesting new results have emerged, or have been reported in greater detail, including the observation of synchronization of switchings between the stable states of a Brownian particle in a bistable optical trap [2], prediction and observation of SR in an underdamped noise-driven monostable system [3], observation of SR in a bistable SQUID loop [4] and in a crayfish mechanoreceptor [5], observation of SR in systems with coexisting periodic attractors $[6,7]$, theory of SR for a quantum two-level system with ohmic dissipation in the context of conduction fluctuations in a mesoscopic wire [8] and observation of the periodic modulation of the conductance in response to modulation of the temperature or electric field [9], theory and observation of SR for a monostable system with a cyclic variable [10], SR in a tunnel diode [11], and noise-enhanced heterodyning in bistable systems [12], a phenomenon closely related to SR.

Stochastic resonance was first discovered [13]-[15] in searching for an explanation of the earth's ice-age cycle (see Section 2A, below), and then for a few years the analysis was concentrated on SR in simple symmetric bistable systems. More recently the context of SR within the larger scientific enterprise has become clear. It is the main aim of this paper to explain how SR relates to standard statistical physics, to point out that the phenomenon becomes analytically tractable when treated through the traditional methods, in particular linear response theory, and to emphasize the close relationship of SR to earlier results in condensed matter physics. The paper is not intended to be a review in the conventional sense, and neither have we attempted to cite every paper published on SR. Rather, we wish to propose a perspective in which SR can be seen to take its place with other phenomena in physics.

Section 2 sets the scene by reviewing major events in the relatively recent history of the subject, i.e. since the term stochastic resonance was introduced. In Section 3, we go back in time to consider the prehistory of SR, showing how linear response theory (LRT) provides the simplest and most natural way of approaching the phenomenon under most conditions, and discussing some precursors of SR in condensed matter physics. There are a number of interesting SR phenomena that lie outside the range of linear response so that LRT is inapplicable, however. They can nonetheless still be treated through application of standard methods of statistical physics: some examples are considered in Section 4. Finally, in Section 5, we draw conclusions and look to the future of SR.

\section{An Historical Overview}

For historical reasons, the term "stochastic resonance" is used for the two distinct sorts of effects that arise in fluctuating systems driven by a periodic force $A$ cos $\Omega t$ : (i) fluctuationmediated periodic modulation of the populations of the coexisting stable states, in bistable systems [13]-[15], and (ii) the increase, with increasing fluctuation intensity, of the periodic signal in the system [13], and of the signal-to-noise ratio $R[16,17]$ - a phenomenon by no 
means limited to bistable systems [3, 10]. We briefly review the origins of the idea of SR in Sec. 2A, and then in Sec. 2B we describe the discovery of SR in a ring laser and how it resulted in an explosion of interest leading to the observation of SR in different types of systems.

\section{A. Ice-ages Prelude}

The term "stochastic resonance" was introduced by Benzi et al. [13] in the context of the theory of ice ages. By the mid-seventies it had been established that the data on the oxygen isotopic composition (relative amount of the ${ }^{18} \mathrm{O}$-isotope) of planktonic foraminifera, and some other independent geological data revealing climatic changes (the ice ages), display periodicity, with a period of $\sim 100,000$ years [18]. This period is close to that of the variations of the eccentricity of the earth's orbit. As a result of the latter the power of solar radiation incident on Earth (averaged over a period long compared to a year) varies correspondingly, and it was hypothesized that this might be responsible for the global climatic changes [19].

A simple model that describes such effects is one in which the climate is described by a single variable, which might be thought of as a global earth temperature $T_{e}$, the equation for $T_{e}$ being the energy balance equation (cf. Refs. [14, 15])

$$
C_{e} \frac{d T_{e}}{d t}=\mu P\left[1-\alpha\left(T_{e}\right)\right]-\tilde{\sigma} T_{e}^{4}
$$

Here, $P$ is the average power of the incoming solar radiation, $C_{e}$ is the thermal capacity of the Earth, $\alpha(T)$ is the average albedo, and $\tilde{\sigma}$ is the average renormalized Stefan constant that describes cooling of the Earth via infrared emission. The parameter $\mu$ characterizes the eccentricity of the Earth's orbit: it depends on time periodically with the period $2 \pi / \Omega=10^{5}$ years,

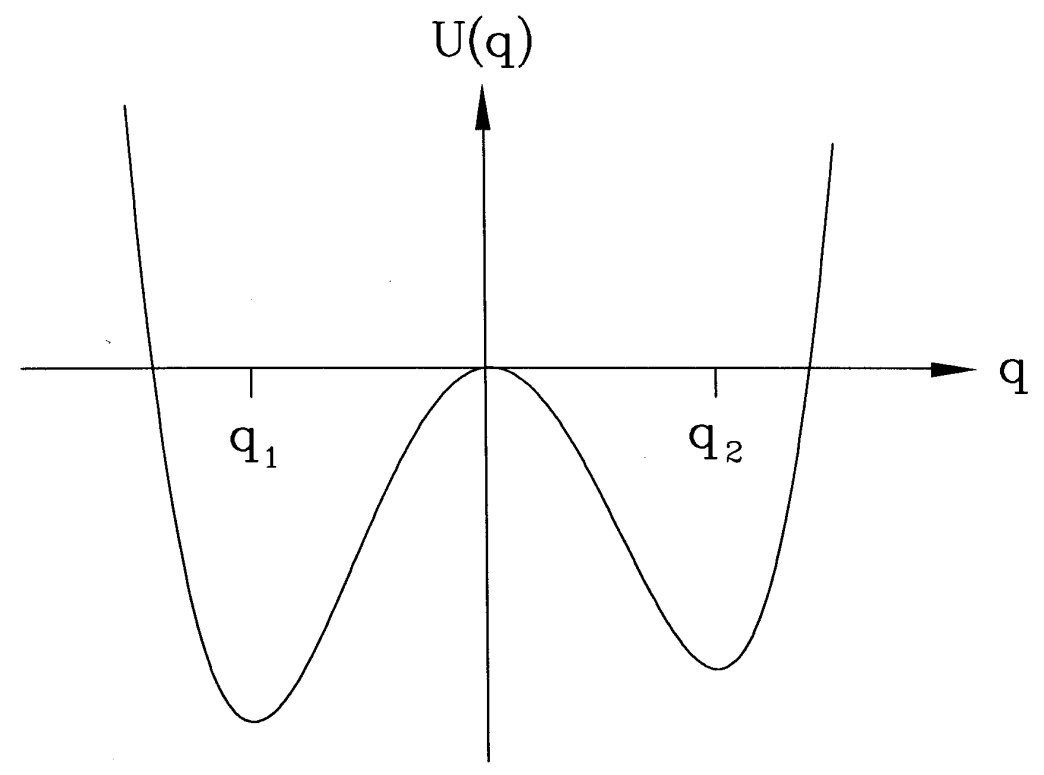

Figure 1: The double-well potential $U(q)$. The minima $q_{1}, q_{2}$ correspond to the stable states of a system with the dynamical variable $q$. In the model of climate (1) $q$ is the effective temperature of the Earth $T_{e}$, and $U\left(T_{e}\right)=\Phi\left(T_{e}\right)$. 


$$
\mu \equiv \mu(t)=1+A \cos \Omega t
$$

Eq.(1) can be rewritten in the form of an equation of motion of an overdamped particle with the coordinate $T_{e}$ in a potential $\Phi\left(T_{e}\right)$ :

$$
\frac{d T_{e}}{d t}=-\frac{\partial \Phi}{\partial T_{e}}, \quad \Phi\left(T_{e}\right)=\int^{T_{e}} d T_{e}^{\prime} C_{e}^{-1}\left(-\mu P\left[1-\alpha\left(T_{e}^{\prime}\right)\right]+\tilde{\sigma} T_{e}^{\prime 4}\right) .
$$

The stable states of the system correspond to the minima of $\Phi\left(T_{e}\right)$ (cf. Fig.1). The model (1) can be relevant to the problem of the ice ages provided there are two stable states coexisting for $\mu=1$ : one with the temperature $T_{e}=T_{e 2}$ for which the Northern Hemisphere is mostly free from ice, and the other with the temperature $T_{e}=T_{e 1}$ for which a substantial part of the Northern Hemisphere is covered by ice. In these two states the reflectivity of the Earth, i.e., the value of the parameter $\alpha$ in (1), is different, and therefore they are both self-consistent (when $T_{e}=T_{e 2}>T_{e 1}$ there is no ice, less energy is reflected, and thus the temperature is higher, whereas for $T_{e}=T_{e 1}<T_{e 2}$ the ice is in position, hence more energy is reflected, and the temperature $i s$ lower; we notice that the difference $T_{e 2}-T_{e 1} \sim 10 \mathrm{~K}$ ).

In the model (1), switching between the states occurs provided the amplitude $A$ of the modulation of the parameter $\mu$ is large enough, so that for $|\mu-1| \leq A$ the system has only one stable state. In other words, when $\mu$ as given by (2) varies periodically in time, the potential $\Phi$ changes so that each of the two wells disappears in turn, and the system is then forced to switch to the other one. However, estimates show that the modulation amplitude $A$ related to the oscillations of the eccentricity of the Earth's orbit is not large enough to provide such a deterministic switching.

It was suggested by C. Nicolis and by Benzi et al. $[14,15]$ that the periodic modulation of the populations of the wells can be mediated by fluctuations. These fluctuations, which have relatively short correlation times, result from atmospheric and oceanic circulation, volcanic eruptions (dust in the atmosphere can change the albedo noticeably, and for quite a long time), etc. In the simplest model they can be assumed to be a white noise driving the global temperature, in which case Eqs.(1), (1a) take the form

$$
\dot{q}=-\frac{\partial U}{\partial q}+\xi(t), \quad\langle\xi(t)\rangle=0, \quad\left\langle\xi(t) \xi\left(t^{\prime}\right)\right\rangle=2 D \delta\left(t-t^{\prime}\right)
$$

with

$$
q \equiv T_{e}, \quad U(q)=\Phi(q),
$$

where $D$ is the characteristic noise intensity. It is assumed to be small so that the fluctuations of $q$ (i.e., of $\left.T_{e}\right)$ about the stable values $q_{1,2} \equiv T_{e 1,2}\left(U^{\prime}\left(q_{1,2}\right)=0, U^{\prime \prime}\left(q_{1,2}\right)>0\right.$, cf. Fig.1) are small compared to $q_{2}-q_{1}$. Nevertheless, although the noise is weak on the average, there can occur, occasionally, outbursts large enough to cause switchings between the stable states. The probability $W_{n m}$ of a switching from the $n$th to the $m$ th state for a white-noise driven system was found by Kramers [20] to be of the activation type,

$$
W_{n m}=\pi^{-1}\left[U^{\prime \prime}\left(q_{n}\right)\left|U^{\prime \prime}\left(q_{s}\right)\right|\right]^{1 / 2} \exp \left(-\Delta U_{n} / D\right), \quad \Delta U_{n}=U\left(q_{s}\right)-U\left(q_{n}\right)
$$

Here, $\Delta U_{n}$ is the depth of the $n$th well of the potential $U(q)$ measured relative to the value $U\left(q_{s}\right)$ of $U(q)$ at its local maximum $q_{s}$ between the minima of $U(q)$ at $q_{1}$ and $q_{2}$ $\left(U^{\prime}\left(q_{s}\right)=0, U^{\prime \prime}\left(q_{s}\right)<0\right.$, see Fig. 1$)$. 
If for $\mu=1$ the depths $\Delta U_{1}$ and $\Delta U_{2}$ are nearly equal, as they are assumed to be in the model of the climate (1), the populations of the stable states vary at random in time, and their average values $w_{1}, w_{2}$ are of the same order of magnitude:

$$
w_{1} / w_{2}=W_{21} / W_{12} \propto \exp \left[\left(\Delta U_{1}-\Delta U_{2}\right) / D\right]
$$

Let us assume now that the potential is slowly modulated in time so that $\Delta U_{1}$ and $\Delta U_{2}$ oscillate in counterphase, and for a half of the period $2 \pi / \Omega$ one of the potential wells is relatively deeper, while for the other half of the period it is shallower. Then the average values of the populations $w_{1,2}$ will oscillate in time as well, i.e., there will occur periodic modulation of the populations for a modulation strength that is much less than that needed to change the potential from a double-well to a single-well. So, a periodic redistribution of the system over the stable states in response to a periodic forcing comes about via noise. It was this interesting idea that was suggested in $[14,15]$ as a plausible explanation of the periodicity of the ice ages.

We notice that, in the very first paper where the term "stochastic resonance" was introduced, [13] an interesting question was raised: is it possible for the noise-mediated modulation of the populations to be so strong that the instantaneous values of the populations, and not just their ensemble-averaged values, depend on time periodically? If so, what are the necessary conditions? Obviously, such a periodicity would arise in the absence of noise for sufficiently strong driving but, at first glance, for a weak periodic driving in the presence of noise the transitions between the states occur at random, although with a tendency towards periodicity. It turns out, however, that even for a weak driving, provided that the driving amplitude exceeds the properly scaled noise intensity and that the modulation frequency is small enough, the redistribution over the states is nearly periodic in time: see Sec. $4 \mathrm{~A}$.

\section{B. SR in a Ring Laser}

The observation of SR in a bidirectional ring laser, reported by McNamara, Wiesenfeld, and Roy [17], played an important role in attracting the attention of the scientific community to SR. A bidirectional ring laser is bistable: it can generate modes that propagate either clockwise or anticlockwise. With the aid of a modulator (an intracavity acousto-optical modulator) it was possible to switch the operation between the modes. The modulator was driven by a broad-band (quasi-white) noise and by a sinusoidal voltage, and the intensity of the radiation of the laser in one direction was detected. In the absence of the periodic modulation this intensity was fluctuating in time at random. When the sinusoidal voltage was applied in addition to the noise there occurred a $\delta$-shaped spike in the power spectrum of the intensity at the frequency $\Omega$ of the voltage (there were also spikes at the overtones). Not only was the intensity of this spike found to increase with increasing intensity of the input noise, but, quite counter-intuitively, the ratio $R$ of the former to the height of the power spectrum at the frequency $\Omega$ in the absence of the periodic driving (the signal-to-noise ratio) was found to increase, too, as shown in Fig.2. Similar behaviour had already been observed by Fauve and Heslot [16] in analog simulations using a Schmitt trigger as the bistable system.

The theory of SR considered in [17] and also in the paper by McNamara and Wiesenfeld [21] was based on the balance equation for the average populations of the coexisting stable states of a noise-driven bistable system:

$$
\dot{w}_{1}(t)=W_{21}(t) w_{2}(t)-W_{12}(t) w_{1}(t), \quad w_{1}(t)+w_{2}(t)=1
$$




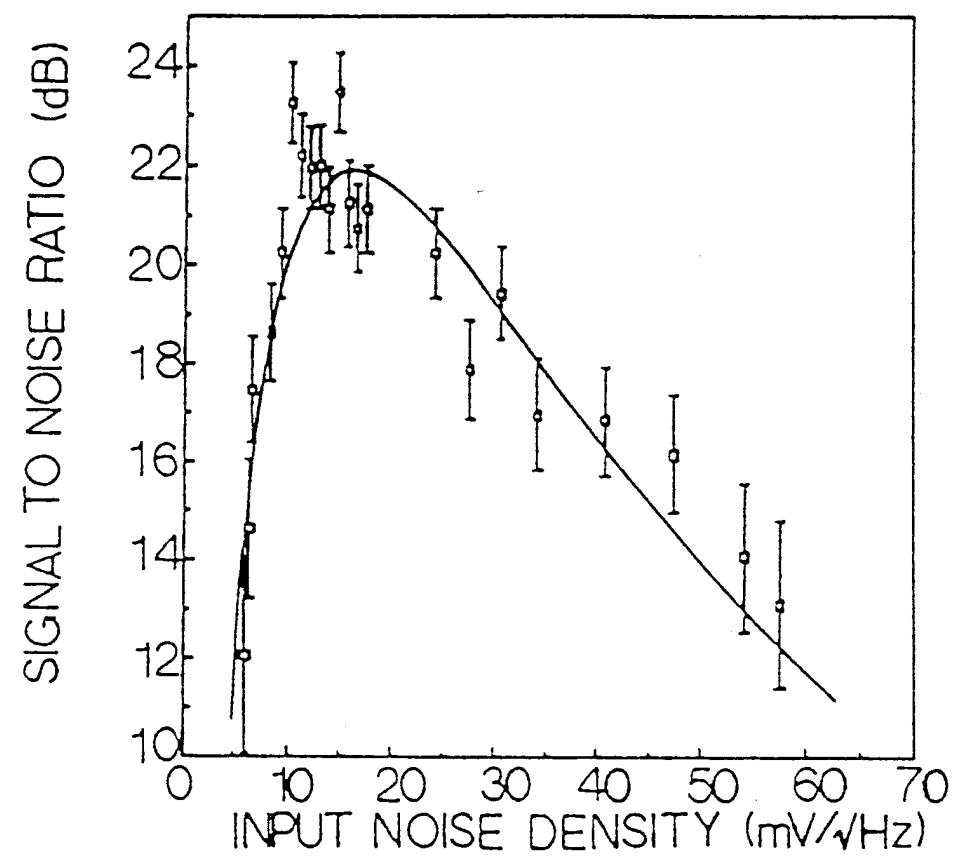

Figure 2: Signal-to-noise ratio vs noise intensity as observed for a ring laser in [17].

The transition probabilities $W_{n m}(t)$ were evaluated in the adiabatic approximation: i.e. it was assumed that the frequency $\Omega$ of the sinusoidal driving was small compared to the reciprocal relaxation time of the system. In this case the probability $W_{n m}(t)$ is determined by the instantaneous value of the potential well depth $\Delta \tilde{U}_{n}(A \cos \Omega t)$ where $\Delta \tilde{U}_{n}(A)$ is the depth of the $n$th potential well in the potential tilted by the external force, $U(q)-A q$. For small enough amplitude of the force only linear terms need be retained in the potential well depth. Using Eq.(4), one can then write $W_{n m}(t)$ in the form [22]:

$$
W_{n m}(t)=W_{n m}^{(0)} \exp \left(g_{n} \cos \Omega t\right), \quad g_{n}=\tilde{g}_{n} A / D, \quad \tilde{g}_{n}=-\left[\frac{\partial \Delta \tilde{U}_{n}(A)}{\partial A}\right]_{A=0}
$$

where $W_{n m}^{(0)}$ is the value of the transition probability in the absence of the force.

It is evident from Eq.(7) that the parameter which determines the effect of the driving is proportional to the ratio of the amplitude of the force $A$ to the noise intensity $D$. Thus, in agreement with what was said above, for small $D$ we can have strong effects even for comparatively small $A$. Moreover, if $A$ is so small that $\left|g_{1,2}\right| \ll 1$, the probabilities $W_{n m}(t)$ can be expanded in $g_{n}$ and Eq.(6) can then be solved analytically. To first order in $\left|g_{1,2}\right|$ the dependence of the populations $w_{1,2}(t)$ on time is sinusoidal.

The solution is particularly simple in the important case of a symmetric potential $U(q)$, for which $\Delta U_{1}=\Delta U_{2}, g_{1}=-g_{2}$. An important byproduct of this solution is an interesting behaviour of the spectral density of fluctuations $Q(\omega)$ of the coordinate of the system $q(t)$ :

$$
Q(\omega)=\lim _{\tau \rightarrow \infty}(4 \pi \tau)^{-1}\left|\int_{-\tau}^{\tau} d t q(t) \exp (i \omega t)\right|^{2}
$$

If the coordinate $q(t)$ is approximated by the sum of its values in the stable states $q_{1,2}$ 
weighted by the populations $w_{1,2}(t)$ (the two-state approximation), then, because of the sinusoidal dependence of the populations on time a $\delta$-shaped spike occurs in $Q(\omega)$ at the frequency $\omega=\Omega$. The ratio of its intensity (area) to the value of the power spectrum $Q^{(0)}(\Omega)$ in the absence of the driving, i.e. the signal-to-noise ratio $R$, is given by the expression $[17,21]$ :

$$
R=\frac{\pi}{4} g^{2} W^{(0)}, \quad g \equiv g_{1}=-g_{2}, \quad W^{(0)} \equiv W_{12}^{(0)}+W_{21}^{(0)}=2 W_{12}^{(0)}
$$

It follows from (7) and (9) that the signal-to-noise ratio increases exponentially with increasing noise intensity for small enough $D$,

$$
R \propto \exp (-\Delta U / D), \quad \Delta U \equiv \Delta U_{1}=\Delta U_{2}(\Delta U \gg D)
$$

i.e. SR occurs. As mentioned above, it is this amazing result that has stimulated so much interest in the phenomenon of stochastic resonance among physicists, biologists, and engineers. In addition to ring lasers, optically trapped Brownian particles, and SQUIDs referred to above, SR has been observed in various analog electronic circuits (see Refs. [16, 6, 7], [23]-[25]), in a passive optically bistable system [26] and in a bistable laser with saturable absorber [27], in a magnetoelastic ribbon [28], in a hybrid device that included an ESR cavity [29], in single-domain uniaxially anisotropic magnetic particles [30], and in a magnetoresistive oscillator [31]. It has also been proposed [32, 33] that $\mathrm{SR}$ may be relevant to the transmission of information by sensory neurons. SR has been shown to occur in models of excitable neurons [34], and the effects on SR of neuron coupling in a neural network have been analyzed [35].

\section{Linear Response Theory and the Prehistory of SR}

\section{A. Linear response Theory}

A simple theoretical approach to SR that makes it possible to place the phenomenon in context within statistical physics and condensed matter physics, and to relate it to what had been done in these areas before, is based on linear response theory (LRT). According to LRT, if a system with a coordinate $q$ is driven by a weak force $A \cos \Omega t$ (the addition to the Hamiltonian function of the system is of the form of $-A q \cos \Omega t$ ), there arises a small periodic term in the ensemble-averaged value of the coordinate, $\delta\langle q(t)\rangle$, oscillating at the same frequency $\Omega$ and with amplitude $a$ proportional to that of the force [36]:

$$
\begin{gathered}
\delta\langle q(t)\rangle=a \cos (\Omega t+\phi) \equiv \operatorname{Re}\left[\chi(\Omega) A e^{-i \Omega t}\right], \quad A \rightarrow 0 \\
a=A|\chi(\Omega)|, \quad \phi=-\arctan [\operatorname{Im} \chi(\Omega) / \operatorname{Re} \chi(\Omega)]
\end{gathered}
$$

The quantity $\chi(\Omega)$ here is the susceptibility of the system. Eq.(10) holds for dissipative and fluctuating systems that do not display persistent periodic oscillations in the absence of the force $A \cos \Omega t$ and where the correlations of fluctuations decay in time. In the more general case of a system performing phase-locked oscillations with a period $2 \pi / \omega_{F}$ (this case is of particular interest for systems driven by strong periodic fields with a frequency $\omega_{F}$, e.g., by laser radiation) the linear response is described by the expression 


$$
\delta\langle q(t)\rangle=\operatorname{Re} \sum_{k=-\infty}^{\infty} \chi^{(k)}(\Omega) A \exp \left[i\left(k \omega_{F}-\Omega\right) t\right], \quad A \rightarrow 0
$$

In this case a weak force gives rise to vibrations not only at its own frequency, but also at the combination frequencies $\left|\Omega \pm k \omega_{F}\right|$, and $\chi_{k}(\Omega)$ are the corresponding susceptibilities.

The function $\chi(\Omega)$ (or the functions $\chi^{(k)}(\Omega)$ ) contains all information on the response of the system to a weak driving force. It gives both the amplitude of the signal, $a$, and its phase lag with respect to the force, $\phi$ (or partial amplitudes and phase lags for the vibrations at the combination frequencies). In fact, Eqs.(10), (11) still hold even if the force is of a more general nature than just an "additive" coordinate-independent force described by the extra term $-A q \cos \Omega t$ in the Hamiltonian. In particular, the force can be coordinate-dependent (a multiplicative force), or it can be the intensity of the noise driving the system (e.g., the temperature, if the noise is of thermal origin) that is modulated periodically. In any case, if the amplitude of the modulation is weak enough, the response of the system is linear and is described by (10), (11). The onset of SR in response to a modulation of the noise intensity (temperature) [37] has been investigated in [8] and [9]. Note that LRT is equally applicable to "non-traditional" SR-displaying systems such as integrate-and-fire neurons [38] and threshold detectors [39].

The periodic terms (10),(11) induced by the force give rise to $\delta$-shaped spikes in the spectral density of fluctuations $(\mathrm{SDF}) Q(\omega)(8)$ at the frequency of the force $\Omega$ (and at the combination frequencies $\left.\left|\Omega \pm k \omega_{F}\right|\right)$. The intensity (i.e., the area) of these spikes is equal to one fourth of the squared amplitude of the corresponding vibrations, i.e., to $\frac{1}{4} A^{2}|\chi(\Omega)|^{2}$, or to $\frac{1}{4} A^{2}\left|\chi^{(k)}(\Omega)\right|^{2}$. The signal-to-noise ratio $R$ is thus expressed in terms of the susceptibility as

$$
R=\frac{1}{4} A^{2}|\chi(\Omega)|^{2} / Q^{(0)}(\Omega) \quad(A \rightarrow 0),
$$

and for periodically oscillating systems the signal-to-noise ratio $R^{(k)}$ at the combination frequency $\left|\Omega-k \omega_{F}\right|$

$$
R^{(k)}=\frac{1}{4} A^{2}\left|\chi^{(k)}(\Omega)\right|^{2} / Q^{(0)}\left(\left|\Omega-k \omega_{F}\right|\right) \quad(A \rightarrow 0)
$$

Therefore, the evolution of the susceptibility and of $Q^{(0)}(\omega)$ with varying noise intensity $D$ show immediately whether or not SR (understood as an increase of the signal-to-noise ratio with increasing $D$ in a certain range of $D$ ) is to be expected at a given frequency.

Describing SR in terms of the susceptibility is particularly advantageous for systems that are in thermal equilibrium or in quasi-equilibrium. In this case the susceptibility can be expressed immediately in terms of the $\operatorname{SDF} Q^{(0)}(\Omega)$ in the absence of periodic driving via the fluctuation-dissipation relations [36]:

$$
\operatorname{Im} \chi(\omega)=\frac{\pi \omega}{T} Q^{(0)}(\omega), \quad \operatorname{Re} \chi(\omega)=\frac{2}{T} \mathrm{P} \int_{0}^{\infty} d \omega_{1} Q^{(0)}\left(\omega_{1}\right) \frac{\omega_{1}^{2}}{\omega_{1}^{2}-\omega^{2}}
$$

where $\mathrm{P}$ implies the Cauchy principal value and $T$ is the temperature in energy units. It follows from (12), (14) that the onset of SR can be predicted from purely experimental data on the evolution of the SDF of a system with temperature without assuming anything at all about the equations that describe its dynamics, i.e. for a system treated as a "black box". 
The relevance of this approach to SR has been demonstrated unambiguously in analog experiments on electronic systems simulating Brownian motion in a bistable [24] and in a monostable [3] potential. In [24] the signal-to-noise ratio for quasithermal fluctuations of a particle with inertia and friction fluctuating in a symmetric double-well potential was (i) measured directly, with a weak periodic force applied to a system, and (ii) calculated from experimental measurements of the power spectrum in the absence of periodic forcing, using Eqs.(12),(14). The dependence of $R$ on the noise intensity exhibited the reversed$N$ shape typical of SR in continuous systems (cf. Figs.5, 7 below), and the results of these two independent experiments were in a perfect qualitative and satisfactory quantitative agreement with each other. The data obtained in [3] show that, contrary to what had been commonly accepted, a noise-induced increase of the signal in a system does not require that it be a bistable one: the effect can arise in monostable systems as well. The particular mechanism explored [3] is based on the fact that the frequency of vibrations in a nonlinear system depend on their amplitude (energy). By varying the temperature of the system (the noise intensity) one varies the distribution of the system over the energy, and hence over the frequency. It is possible therefore to "tune" the system, and thus to increase the response at an appropriate frequency. The strong and rather interesting temperature dependence of the spectral density of the fluctuations $Q^{(0)}(\omega)$ of underdamped systems was reviewed in Ref. [40]. Recent results obtained for a special class of underdamped systems where the dependence of the eigenfrequency of the vibrations on the amplitude is nonmonotonic - the upper curve in Fig. 3(b) refers to a system of this sort - are reported in [41]; it is in this case that the noise-induced increase of the signal-to-noise ratio, not of the signal only, was observed in an underdamped system [3].

\section{B. Precursors of Stochastic Resonance in Condensed Matter Physics}

To the best of our knowledge, analytical results for the susceptibility of a fluctuating symmetrical system with two coexisting stable states, which traditionally has been of primary interest in the context of SR, were first obtained by Debye [43]. Debye analyzed the dielectric response of polar molecules in a solid (in ice - a material that is somehow magic for SR!). He assumed that a molecule can switch between two equivalent positions within a unit cell, and that in these positions the dipole moment of the molecule is pointing in opposite directions. The expression for the transition probability $W_{n m}$ he used was equivalent to Eq.(7), with $g_{n}=-\mathbf{E} \mathbf{d}_{n} / T$ where $\mathbf{E}$ is the amplitude of the electric field and $\mathbf{d}_{n}$ is the dipole moment in the $n$th position $\left(n=1,2 ; \mathbf{d}_{1}=-\mathbf{d}_{2}\right)$; he linearized $W_{n m}$ in $\mathbf{E} \mathbf{d}_{n} / T$ (however, he did not specify the form of the transition probabilities $W_{n m}^{(0)}$ in the absence of the external field).

The well-known expression for the susceptibility Debye derived was, in the present notation, of the form

$$
\chi_{\mathrm{D}}(\Omega)=\frac{d_{1}^{2}}{T} \frac{W^{(0)}}{W^{(0)}-i \Omega}, \quad W^{(0)} \equiv W_{12}^{(0)}+W_{21}^{(0)}=2 W_{12}^{(0)} .
$$

This expression made it possible to explain the experimental data on the dispersion of the real part of the dielectric constant of ice. It is straightforward to see from the fluctuationdissipation relations (14) that the signal-to-noise ratio $R$ that follows from $(12),(15)$ is precisely of the form (9) (cf. [24, 42]).

In the context of condensed-matter physics, the quantity of special interest is often the phase shift between the force and the signal, since it is the phase shift that determines the absorption of the energy from the force, in particular from the electromagnetic field in 


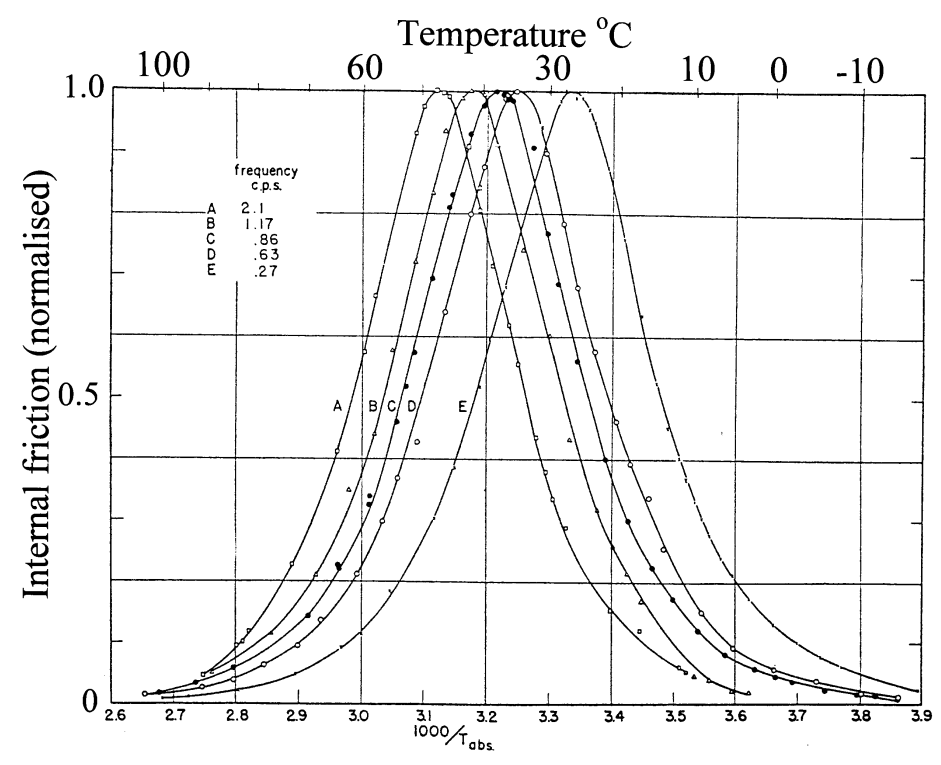

Figure 3: Peaks of internal friction (normalized phase lag $-\phi$ ) vs temperature due to Snoek relaxation in an Fe-C alloy; the curves $A$ to $E$ correspond to the frequencies $2.1 ; 1.17 ; 0.86$; $0.63 ; 0.27 \mathrm{~Hz}$ (after Wert and Zener [45]).

the case considered by Debye. In the symmetrical two-state model with thermally activated transitions between the states the phase shift $\phi$ as given by (10),(15) decreases monotonically with increasing temperature $[43,14,21]$ :

$$
(\phi)_{\text {two-state }}=-\arctan \left(\Omega / W^{(0)}\right)
$$

The phase shift is one of the characteristics used to describe the elastic properties of solids: in this case the force is stress, the signal is strain, and the phase lag is referred to as internal friction [44]. For finite frequency of the stress there arises a phase shift between the stress and the strain, even though the stress is linear in the strain (and thus reversible). In some metal alloys internal friction displays a strong nonmonotonic temperature dependence as shown in Fig.3 taken from [45]. A simple mechanism of this dependence for body-centered cubic metals with interstitial impurity atoms was suggested by Snoek [46]. He assumed that an impurity occupies one of the equivalent interstitial positions in an elementary cell thus forming an elastic dipole. The dipole can reorient as a result of thermal fluctuations. Uniaxial stress breaks the symmetry, like an electric field in the case of electric dipoles, and the response to the stress is given basically by Debye's theory, slightly modified to allow for a different number of equivalent stable states.

The strain measured experimentally arises as a combination of the strain related to the reorientation of the elastic dipoles and the strain due to the deformation of those cells that are free of impurities. This deformation is characterized by much faster relaxation than the reciprocal reorientation rate $1 / W^{(0)}$ of the dipoles at room temperature. For low temperatures the reorientation rate $W^{(0)}$ is negligibly small, and the strain is equal to that for a crystal with immovable defects and is in phase with the stress (Hooke's law). Therefore the phase shift is equal to zero rather than to $-\pi / 2$ as given by (16). Only for higher $T$ does the reorientation of the elastic dipoles become "switched on" and the term described 


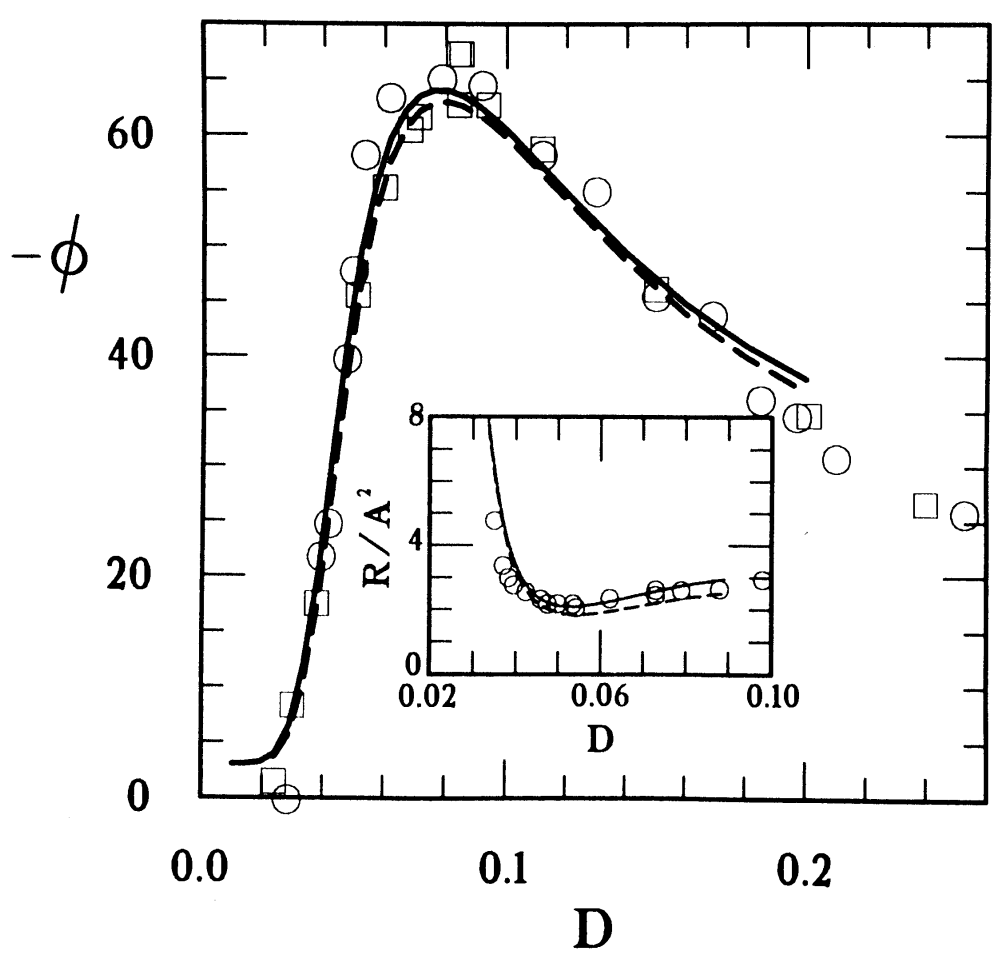

Figure 4: Phase lag $-\phi$ between the coordinate $\langle q(t)\rangle$ of an overdamped Brownian particle oscillating in a potential $U(q)=-\frac{1}{2} q^{2}+\frac{1}{4} q^{4}$ and the force of frequency $\Omega=0.1$ as measured in the electronic experiment; the force amplitude $A=0.04$ (circles) and 0.2 (squares). The solid line represents the theoretical prediction based on LRT [47] (nonlinear corrections do not change this curve strongly for the actual value of $A$ ). The inset shows the normalized signal-to-noise ratio in the region of the minimum in $R$.

by (16) contributes to the phase shift. As a result $|\phi|$ sharply increases with temperature and displays a clearly resolved peak. The position of the peak (see the next subsection) may be used to determine the activation energy for reorientation of the elastic dipoles [44].

\section{Stochastic resonance in continuous dynamical systems}

In many cases, the bistable dynamical systems where SR is investigated are continuous rather than being two-state ones. For such systems, the dependence of the phase lag on the noise intensity (temperature) is similar to that observed for internal friction in anelastic solids. This is clearly seen from a comparison of Fig.3 and Fig.4. In the latter case, the data [47] are from an analog simulation of overdamped Brownian motion (3) in a simple symmetric bistable potential

$$
U(q)=-\frac{1}{2} q^{2}+\frac{1}{4} q^{4} .
$$

The explicit expressions for the phase shift and for the signal-to-noise ratio $R$ of a continuous system (3) for low noise intensities and for low frequency $\Omega$ are of the form:

$$
\phi=-\arctan \left[\left(\Omega / \Omega_{r}\right)\left(\Omega_{r}^{2} W^{(0)}+\Omega^{2} D\right) /\left(\Omega_{r} W^{(0) 2}+\Omega^{2} D\right)\right]
$$




$$
R=\frac{\pi A^{2}}{4 D^{2}}\left(\Omega_{r}^{2} W^{(0) 2}+\Omega^{2} D^{2}\right) /\left(\Omega_{r}^{2} W^{(0)}+\Omega^{2} D\right), \quad \Omega, D \ll \Omega_{r}, \quad W^{(0)} \ll D
$$

where $\Omega_{r} \equiv t_{\mathrm{rel}}^{-1}=U^{\prime \prime}\left(q_{1,2}\right)$ is the reciprocal relaxation time for the intrawell motion (corrections to (18) of the order of $\Omega / \Omega_{r}, W^{(0)} / \Omega_{r}, D / \Delta U$ have been dropped). It is straightforward to see that the maximum of $|\phi|$ as given by (18) occurs at the noise intensity $D_{\max }$ given by the equation

$$
W^{(0)}\left(D_{\max }\right)=\Omega\left(D_{\max } / \Omega_{r}\right)^{1 / 2}
$$

The value of $D_{\max }$ therefore depends on the frequency of the signal $\Omega$, as observed in anelastic solids (cf. Fig.3; the model (3), (17), although similar, is not entirely identical to the one used in the theory of anelastic relaxation, and therefore the peak in Fig. 4 for the dynamical system is much broader the corresponding peak in Fig.3).

The response of a continuous system differs markedly from that of a two-state one, not only in its non-monotonic rather than monotonic variation of $\phi$ with $D$, but also in the variation of its signal/noise ratio with $D$ : for small $D$ the function $R$ decreases rather than increases with increasing $D[21,24]$ (see insert in Fig.4, and also Fig.5 where similar behaviour is demonstrated in a different system). Such behaviour has a simple explanation [24]. For small $D$ the interwell transitions are frozen out: the susceptibility is then determined by the intrawell motion of the system, and is independent of noise, whereas the power spectrum is formed by the fluctuations about the minima of the potential and increases proportional to the noise intensity, so that $R \propto 1 / D$, as seen from (18) for small $W^{(0)}$. The analysis of the position of the local maximum of $R$ vs noise intensity was performed by Fox and $\mathrm{Lu}$ [48].

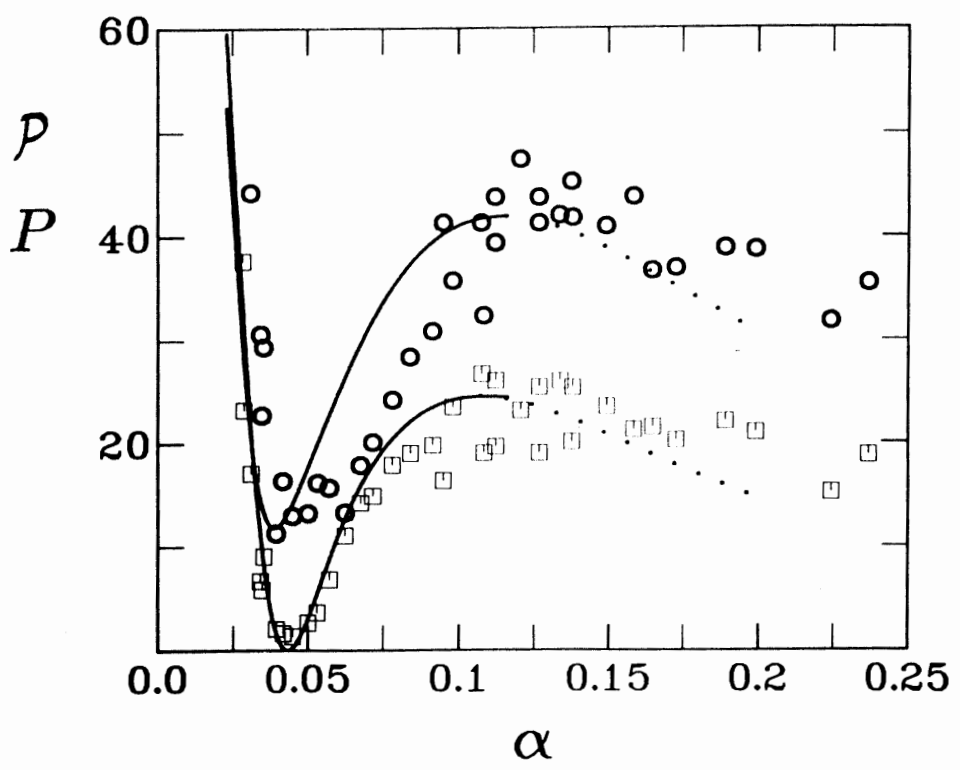

Figure 5: The response of a noise-driven underdamped nonlinear oscillator with coexisting periodic attractors to an extra force $A \cos \Omega t$ (after [7]). The equation of motion of the oscillator for $A=0$ is of the form $\ddot{q}+2 \Gamma \dot{q}+\omega_{0}^{2} q+q^{3}=F \cos \omega_{F} t$. The quantities $P$ and $\mathcal{P}$ represent signal-to-noise ratios at the frequencies $\Omega$ and $2 \omega_{F}-\Omega$. The data refer to the kinetic phase transition range, $\left(\omega_{F}-\omega_{0}\right) / \Gamma=0.236,3 F^{2} / 32 \omega_{F}^{3}\left(\omega_{F}-\omega_{0}\right)^{3}=0.0814$. 
In general, of course, the motion of a bistable continuous system will not be described by the simple model (3) of overdamped Brownian motion in a symmetrical double-well potential. Neither will the noise be white, nor will the system be moving in a static potential. For example, the stable states of interest may be the states of stable periodic vibrations in a strong external force (periodic attractors), as is of interest in the context of optical bistability [49]. Analytic results for the fluctuations and for the response to a weak external force can be obtained [50] provided the noise intensity is small so that the probabilities, per unit time, of fluctuational transitions between the states are very much smaller than the reciprocal intrawell relaxation time, $W_{n m}^{(0)} \ll t_{\text {rel }}^{-1}$, and the fluctuations occur mostly within narrow vicinities of the stable states. The results hold for systems driven by an arbitrary Gaussian noise, where, apart from special cases, the dependence of the transition probabilities on the characteristic noise intensity $\mathcal{D}$ is of the activation type, and in the absence of the additional weak force

$$
W_{n m}^{(0)}=\text { const } \times \exp \left(-\mathcal{R}_{n} / \mathcal{D}\right) .
$$

The activation energy of the escape from the state $n, \mathcal{R}_{n}$, is given by the solution of a variational problem [51]. For certain types of non-white Gaussian noise $\mathcal{R}_{n}$ was found in Refs. [51, 52].

For small enough $\mathcal{D}$ in the case of period-one attractors there are two main contributions to the susceptibilities $\chi^{(k)}(\Omega)$ as defined by Eq.(11) [50]. One comes from the motion close to the stable states where the system spends most of the time. This contribution is given by the sum of the partial susceptibilities $\chi_{n}^{(k)}(\Omega)(n=1,2)$ weighted by the populations of the stable states $w_{n}$. The other contribution, $\chi_{\operatorname{tr}}^{(k)}(\Omega)$, is important in the case where the frequency $\Omega$ of the weak force is small or is close to the frequency $\omega_{F}$ of the strong external force. In this case the weak force modulates the probabilities of the transitions between the states and thus the populations of the states (see below):

$$
\chi^{(k)}(\Omega)=\sum_{n=1,2} w_{n} \chi_{n}^{(k)}(\Omega)+\chi_{\mathrm{tr}}^{(k)}(\Omega), \quad w_{1}=1-w_{2}=W_{21}^{(0)} / W_{12}^{(0)}
$$

The partial susceptibilities $\chi_{n}^{(k)}(\Omega)$ can be easily found from the equations of motion linearized about the stable states in the absence of noise (noise determines the values of the populations $w_{n}$ via the transition probabilities). They display dispersion on the frequency scale $t_{\mathrm{rel}}^{-1}$, and in the range of interest for $\mathrm{SR}, \Omega \ll t_{\mathrm{rel}}^{-1}$ or $\left|\Omega-\omega_{F}\right| \ll t_{\mathrm{rel}}^{-1}$ they are nearly frequency-independent.

The characteristic frequency scale which determines the dispersion of $\chi_{\mathrm{tr}}^{(k)}(\Omega)$ is given by the relaxation rate of the populations, i.e., by $W^{(0)}=W_{21}^{(0)}+W_{12}^{(0)}$. A simple way to obtain $\chi_{\mathrm{tr}}^{(k)}(\Omega)$ for $\Omega \ll \nu_{c}$ or $\left|\Omega-\omega_{F}\right| \ll \nu_{c}\left(\nu_{c}=\min \left(t_{\text {rel }}^{-1}, t_{\text {cor }}^{-1}\right)\right.$, where $t_{\text {cor }}$ is the correlation time of the noise) is based [50] on the fact that the major effect of the additional weak force $A \cos \Omega t$ on the populations of the states comes from the modulation of the activation energies of the transitions between the states $\mathcal{R}_{n}$. For small $\Omega$ one can find this modulation just by evaluating $\mathcal{R}_{n}$ for a system biased by a constant force $A$, i.e., by finding $\mathcal{R}_{n} \equiv \tilde{\mathcal{R}}_{n}(A)$, and then by replacing $A$ by $A \cos \Omega t$. In this case the escape probability can be written in the form similar to $(7)$ : 


$$
W_{n m}(t)=W_{n m}^{(0)} \exp \left(g_{n} \cos \Omega t\right), \quad g_{n}=\tilde{g}_{n} A / \mathcal{D}, \quad \tilde{g}_{n}=-\left[\frac{\partial \tilde{\mathcal{R}}_{n}(A)}{\partial A}\right]_{A=0} \quad\left(\Omega \ll \nu_{c}\right) .
$$

In the case of periodic attractors corresponding to forced vibrations in a strong periodic force $F \cos \left(\omega_{F} t+\phi_{F}\right)$ the additional weak force $A \cos \Omega t$ with $\Omega$ very close to $\omega_{F}$ can be considered as a modulation of the amplitude of the strong force,

$$
\begin{gathered}
F \cos \left(\omega_{F} t+\phi_{F}\right)+A \cos \Omega t=\operatorname{Re} \tilde{F}(t) \exp \left(i\left(\omega_{F} t+\phi_{F}\right)\right) \\
\tilde{F}(t)=F+A \exp \left[i\left(\Omega-\omega_{F}\right) t-i \phi_{F}\right]
\end{gathered}
$$

The activation energies $\mathcal{R}_{n} \equiv \mathcal{R}_{n}(F)$ are independent of the phase $\phi_{F}$, and when the weak force $A \cos \Omega t$ is applied they take on time-dependent values corresponding to the instantaneous value of the amplitude $|\tilde{F}(t)|$, so that

$$
\begin{gathered}
W_{n m}(t)=W_{n m}^{(0)} \exp \left[g_{n} \cos \left(\left(\Omega-\omega_{F}\right) t-\phi_{F}\right)\right], \quad g_{n}=\tilde{g}_{n} A / \mathcal{D}, \\
\tilde{g}_{n}=-\frac{\partial \mathcal{R}_{n}(F)}{\partial F} \quad\left(\left|\Omega-\omega_{F}\right| \ll \nu_{c}, \omega_{F}\right)
\end{gathered}
$$

Eqs. (21), (22) can be inserted into Eq.(6) for the populations. For small amplitudes $A$, when $\left|g_{n}\right| \ll 1$, one can expand the transition probabilities in $g_{n}$. Terms linear in $g_{n}$ are sinusoidal in time, and so also are the corresponding terms in the populations $w_{1,2}(t)$. If we write the expression for the coordinate in the $n$th period-one attractor as

$$
q_{n}(t)=\sum_{k} q_{n}^{(k)} \exp \left(i k \omega_{F} t\right)
$$

then the expression for the susceptibility $\chi_{\operatorname{tr}}^{(k)}(\Omega)$ for $\left|\Omega-\omega_{F}\right| \ll \nu_{c}$ is of the form (cf. [50])

$$
\chi_{\mathrm{tr}}^{(k)}(\Omega)=-\frac{W_{12}^{(0)} W_{21}^{(0)}}{W^{(0)}} \frac{\tilde{g}_{1}-\tilde{g}_{2}}{\mathcal{D}} \frac{q_{1}^{(k-1)}-q_{2}^{(k-1)}}{W^{(0)}-i\left(\Omega-\omega_{F}\right)} e^{i \phi_{F}}
$$

The equation for the susceptibility with respect to a low-frequency force, $\Omega \ll \nu_{c}$, is very similar:

$$
\chi_{\operatorname{tr}}(\Omega)=-\frac{W_{12}^{(0)} W_{21}^{(0)}}{W^{(0)}} \frac{\tilde{g}_{1}-\tilde{g}_{2}}{\mathcal{D}} \frac{q_{1}^{(0)}-q_{2}^{(0)}}{W^{(0)}-i \Omega} \quad\left(\Omega \ll \nu_{c}\right) .
$$

It can easily be seen that in the symmetrical case, $W_{12}^{(0)}=W_{21}^{(0)}, \tilde{g}_{1}=-\tilde{g}_{2}, q_{1}^{(0)}=-q_{2}^{(0)}$, this expression goes over into Debye's result (15).

Note that, for a simple model of overdamped Brownian motion in the bistable potential (3), the expressions for the susceptibility (20), (23a) (and also the explicit form of the partial susceptibility $\chi_{n}(\Omega)$ ) can be obtained at low noise intensities directly from an analysis of the eigenvalues and eigenfunctions [53] of the Fokker-Planck equation, both in the case of a symmetric [54](a) and an asymmetric [54](b) potential. Alternatively, one can evaluate the power spectrum in the absence of periodic driving $Q^{(0)}(\omega)$ and use the fluctuation-dissipation 
relations (14). The function $Q^{(0)}(\omega)$ was found by Hänggi and Thomas [55]. However, they failed to get the correct expression for the term $\chi_{\mathrm{tr}}$ in the susceptibility which is responsible for the onset of SR. A detailed numerical analysis of the Fokker-Planck equation for the system (3), (17) is [56] in full agreement with the analytic results given above, and in particular with those for the phase shift shown in Fig. 4. Note also some earlier numerical work on the Fokker-Planck equation for periodically driven bistable systems [57].

From (21) - (23) (cf. [50]) it is clear : (i) that the susceptibility due to the transitions between the states increases, for finite frequency, exponentially sharply with noise intensity $\mathcal{D}$ in the range of very small $\mathcal{D}$; (ii) that this susceptibility is greatest within a frequency range that is extremely narrow compared with the characteristic inverse relaxation time $t_{\text {rel }}^{-1}$; (iii) that the susceptibility is proportional to the reciprocal noise intensity, which is why it can become large, and (iv) that it becomes large only within the narrow range of the system parameters for which $\mathcal{R}_{1} \approx \mathcal{R}_{2}$, and thus the transition probabilities, $W_{12}^{(0)}$ and $W_{21}^{(0)}$, and the populations, $w_{1}$ and $w_{2}$, are of the same order of magnitude (the range of the kinetic phase transition). All of these features have been observed in experiments, and are immediately related to the onset of $\mathrm{SR}$ in bistable systems. In particular, the feature (iv) shows that $\mathrm{SR}$ in bistable systems is a kinetic phase transition effect. Some results demonstrating SR in a system with periodic attractors, obtained from an analog electronic experiment [7], are shown in Fig.5. The experimental data (points) exhibit an increase of signal-to-noise ratio both at the frequency of the force $\Omega$ and at the combination frequency $2 \omega_{F}-\Omega$. The onset of a strong signal at the latter frequency is a demonstration of a new phenomenon that arises through the noise-induced interattractor transitions and may be called, using the terms of nonlinear optics, a "giant noise-mediated four-wave mixing". The results are clearly seen to be in a good agreement with the LRT theoretical predictions (curves).

An interesting situation arises if a system displays period-doubling when driven by a strong enough periodic field. If, in addition, the system is driven by a stationary noise, the stationary populations of the two co-existing period-two attractors are the same: the only difference between the attractors is that they are shifted in time by the period of the force $2 \pi / \omega_{F}$ and, because of the symmetry with respect to translation in time by $2 \pi / \omega_{F}$, there is no reason for one of them to be occupied for more of the time than the other. Such a system is therefore a perfect candidate for observing SR and, indeed, SR in an analog electronic circuit that displayed period doubling has been observed recently [6]. Strictly speaking, when the system is driven by a weak force $A \cos \Omega t$ in addition to the strong force $F \cos \omega_{F} t$, persistent vibrations at the frequencies $\left|\Omega \pm \frac{1}{2} \omega_{F}\right|$ do not arise because of the occurrence of transitions between the period two attractors. However, since the lifetime of the attractors (equal to the reciprocal transition probabilities) is large, narrow peaks are to be expected in the power spectrum of the system at $\left|\Omega \pm \frac{1}{2}(2 k+1) \omega_{F}\right|$, with a width $\sim W_{12}$. The increase of the intensity of the peak at $\left|\Omega-\frac{1}{2} \omega_{F}\right|$ with increasing noise intensity as observed in [6] is shown in Fig.6.

Our outline of the history of stochastic resonance would be incomplete if we forbore to mention that, in spite of being so well known in other areas of physics, linear response theory for the first few years after it was applied to SR [24] faced strong arguments and encountered serious problems with gaining recognition in this context. The inapplicability of LRT would imply either that statistical physics as a whole is fatally flawed, or that it does not apply to the systems that display SR. In fact, the two statements are equivalent, because, generally speaking, there is nothing special about the dynamics of the continuous fluctuating bistable 


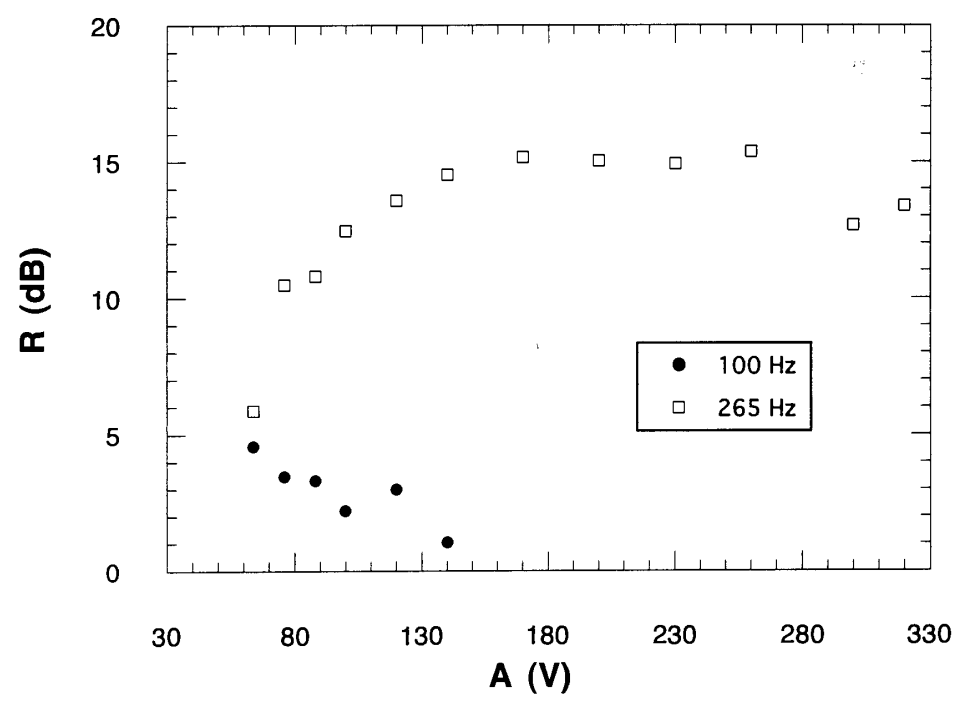

Figure 6: Signal-to-noise ratio for the signals at the frequency of a comparatively weak driving force $\Omega=100 \mathrm{~Hz}$ and at the combination frequency $\frac{1}{2} \omega_{F}-\Omega=265 \mathrm{~Hz}$ vs effective intensity of the chaotic signal driving a system that displays period doubling, as obtained in analog electronic experiments [6].

systems that display SR. We find it unsurprising therefore that the results for SR derived from LRT are in good agreement with the experiments.

We would point out also that a corollary of LRT is that, for small-amplitude signals, the signal-to-noise ratio at the output of a system driven by a stationary Gaussian noise does not exceed that at the input, even if the system displays SR. Indeed, the Fourier components of the noise are statistically independent and the total power of the noise $\Xi(\Omega) d \Omega$ in a small spectral interval $d \Omega$ about the frequency of the signal $\Omega$ is small. The signal-to-noise ratio at the input is given by $\frac{1}{4} A^{2} / \Xi(\Omega)$, whereas that at the output is $\frac{1}{4}|\chi(\Omega)|^{2} A^{2} /\left[|\chi(\Omega)|^{2} \Xi(\Omega)+\right.$ $Q^{\prime(0)}(\Omega)$ ]. The quantity $Q^{\prime(0)}(\Omega)$ gives the value of the spectral density of fluctuations in the system at frequency $\Omega$ as it would be if there was no signal and the spectral components of the noise at frequency $\Omega$ were suppressed, i.e., the power spectrum of the input noise had a hole at frequency $\Omega$. By construction $Q^{\prime(0)}(\Omega) \geq 0$, which proves the statement (In linear systems, on the other hand, which do not mix frequencies, $Q^{\prime(0)}(\Omega)=0$ and the signal-to-noise ratio at the output must be the same as at the input).

Another corollary concerns the problem of the response to an incoherent signal $f_{\text {sig }}(t)$, i.e. to a signal which itself is a narrow-band noise, which was addressed recently in [58]. Again, if this noise is stationary and Gaussian, so that different Fourier-components are statistically independent,

$$
f_{\mathrm{sig}}(t)=\int d \omega \tilde{f}_{\mathrm{sig}}(\omega) \exp (-i \omega t), \quad\left\langle\tilde{f}_{\mathrm{sig}}(\omega) \tilde{f}_{\mathrm{sig}}\left(\omega^{\prime}\right)\right\rangle=\Phi_{\mathrm{sig}}(\omega) \delta\left(\omega+\omega^{\prime}\right)
$$

the response to this signal in the presence of an independent external noise is described by LRT, and for small $\Phi_{\text {sig }}(\omega)$ the addition to the power spectrum of the system

$$
\delta Q(\omega)=|\chi(\omega)|^{2} \Phi_{\operatorname{sig}}(\omega)
$$


This term may increase with the intensity of the "independent" noise provided $|\chi(\omega)|^{2}$ increases in the appropriate frequency range.

\section{Nonlinear Effects in Stochastic Resonance}

Although, as shown above, the LRT approach to SR is immensely fruitful and enables the phenomenon to be described, qualitatively and quantitatively, under a wide range of conditions for small values of $A$, there are other parameter ranges lying beyond the regime of linear response which are also of interest. In one of these, the low-noise limit, analytic results can still be obtained even when $A$ is not particularly small. It is discussed in Section 4A. A second example of an interesting nonlinear regime for which analytic results can be derived, the SR-related phenomenon of noise enhanced heterodying, is discussed in Section $4 \mathrm{~B}$.

\section{A. Nonlinearity in the low-frequency response of a bistable system}

In the publication where the term "stochastic resonance" was originally introduced [13] it was pointed out that the response of a bistable system to external driving can display strong nonlinearity. Indeed, according to $(7),(21),(22)$ the probability $W_{n m}(t)$ of escape from a metastable state $n$ can change strongly when the amplitude $A$ of the driving force, properly weighted, exceeds the noise intensity, i.e., when $\left|g_{n}\right| \gg 1$. For small noise intensities this can occur even when the force is "dynamically small" so that it does not change the number or character of the steady states and nor does it move them significantly in the space of dynamical variables $[22,24,59]$. The nonlinearity is at its strongest for frequencies $\Omega$ very much smaller than the reciprocal relaxation time of the system $t_{\mathrm{rel}}^{-1}$, or for $\Omega$ very close to the frequency of the strong field $\omega_{F}$ in cases where one is present; in what follows we limit ourselves to systems that are not subject to such a strong periodic field, and only the case of small $\Omega$ will be considered.

The mechanism of the nonlinearity for $\left|g_{1,2}\right| \gg 1$ is easily understood because the transition probabilities then vary by orders of magnitude within a period $2 \pi / \Omega$, according to (7). This variation may result in a strong modulation of the populations of the stable states $w_{1,2}(t)$, particularly when the probabilities $W_{12}(t), W_{21}(t)$, depend on time in counterphase, $g_{1} g_{2}<0$ (as in (7)). Indeed, for $\left|g_{1,2}\right| \gg 1$ the transitions from a given stable state $n$ are most likely to occur within a short part of the period $2 \pi / \Omega$ when $W_{n m}(t)$ is close to its maximum. At this time $W_{m n}(t)$ is close to its minimum, for $g_{1} g_{2}<0$. One half-period away from this time, $W_{m n}(t)$ will in turn be close to its maximum, and it is then that transitions to the $n$th state will be most likely to occur.

The population of the $n$th state is at its maximal value, $w_{n>}$, just before $g_{n} \cos \Omega t$ has reached its maximum, and then, after a short time interval where the system is most likely to switch, $w_{n}(t)$ drops to its minimal value, $w_{n<}$. The evolution of $w_{n}(t)$ within this time interval $\delta t_{0}$ is described by the expression $[22,24]$

$$
\begin{gathered}
w_{n}(t) \approx w_{n>} \exp \left(-\int_{t_{0}-\delta t_{0}}^{t} d t^{\prime} W_{n m}\left(t^{\prime}\right)\right),\left|g_{n}\right| \gg 1, g_{1} g_{2}<0,\left|t-t_{0}\right| \ll \pi / \Omega \\
t_{0}=2 \pi k / \Omega \text { for } g_{n}>0, t_{0}=\pi(2 k+1) / \Omega \text { for } g_{n}<0
\end{gathered}
$$




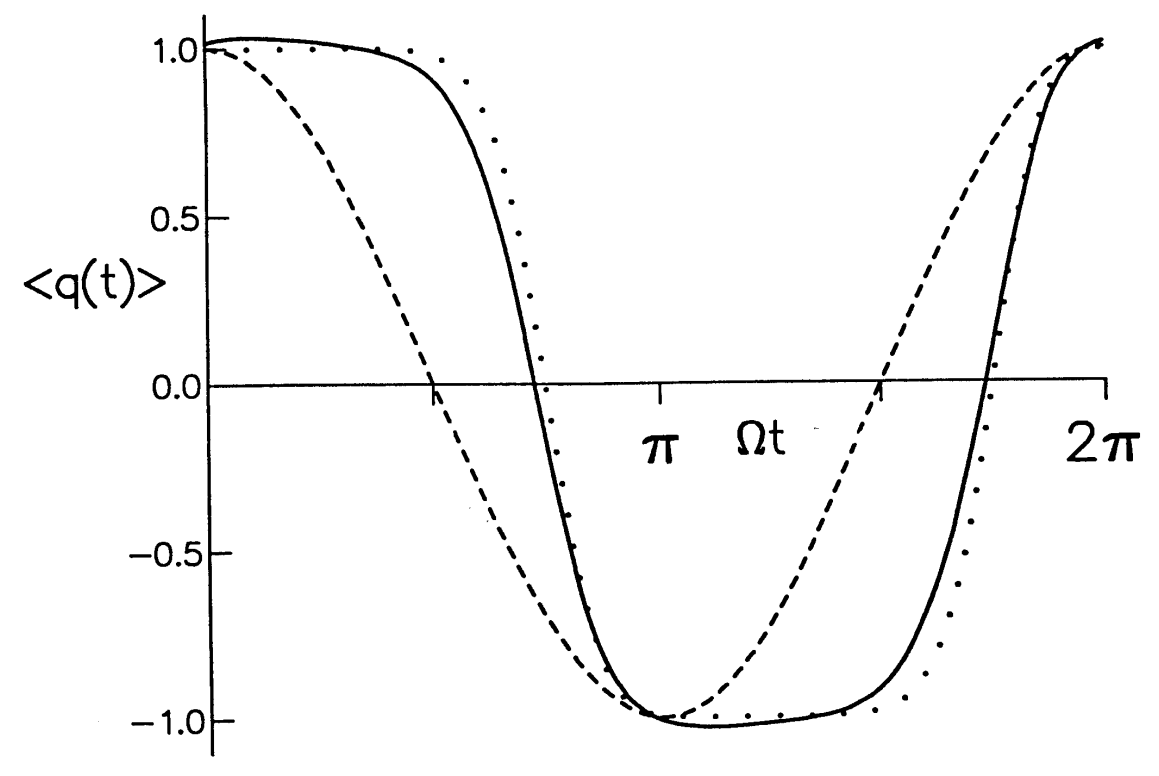

Figure 7: The results of a digital simulation of the ensemble average coordinate of a fluctuating overdamped symmetrical system (3), (17) with $\Omega=3 \times 10^{-4}, A=0.12, D=0.03$ (full curve) compared to the simusoidal force scaled to the same amplitude (dashed curve) (after [22]). The dotted line describes the theory based on (25), (27).

The integral (24) can be evaluated by the steepest descent method, yielding

$$
w_{n}(t) \approx w_{n>} \exp \left(-\lambda_{n}\left[1+\operatorname{erf} \bar{t}_{n}(t)\right]\right), \quad \lambda_{n}=W_{n m}^{(0)} \exp \left(\left|g_{n}\right|\right)\left(2\left|g_{n}\right| \Omega^{2} / \pi\right)^{-1 / 2}
$$

where $\bar{t}_{n}(t)=\left(t-t_{0}\right) / \tau_{n}$. The quantity $\tau_{n} \equiv \Omega^{-1}\left(2 /\left|g_{n}\right|\right)^{1 / 2}$ gives the characteristic time interval over which $w_{n}(t)$ varies from its maximum value $w_{n>}$ to its minimum value $w_{n<}=$ $w_{n>} \exp \left(-2 \lambda_{n}\right)$. We are assuming that $\pi \Omega^{-1} \gg \delta t_{0} \gg \tau_{n}$ in which case the integral (24) is independent of the value of $\delta t_{0}$.

The parameter $\lambda_{n}$ in $(25)$ characterizes $[24,59]$ the nonlinearity of the response. It gives the probability of the transition from the state $n$ over the time $\tau_{n}$ where the transition probability is at its maximal because of the periodic driving.

The values of $w_{n>}, w_{n<}$ can easily be obtained from (25) by noticing that $w_{n>}+w_{m<}=$ $1, \quad n, m=1,2(n \neq m)$. On a time scale coarse-grained over $\tau_{1,2}$ the dependence of the populations $w_{1,2}(t)$ on time takes the form of a square wave, with the jumps between the smaller and larger values occurring every half period of the force. The amplitude of the jumps

$$
\Delta w \equiv \frac{1}{2}\left(w_{1>}-w_{1<}\right) \equiv \frac{1}{2}\left(w_{2>}-w_{2<}\right)=\frac{\sinh \lambda_{1} \sinh \lambda_{2}}{\sinh \left(\lambda_{1}+\lambda_{2}\right)} .
$$

This amplitude is large when both parameters $\lambda_{1}$ and $\lambda_{2}$ are of the order of, or exceed, unity. Obviously, this may occur at small noise intensities provided the frequency $\Omega$ is small enough. On the other hand, if $\lambda_{1}, \lambda_{2}>1.5$ the value of $\Delta w$ exceeds 0.45 , i.e., it is close to the limiting value 0.5. This means, in particular, that for such $\lambda_{1,2}$ the switchings between the states occur nearly every half-period of the force, i.e., the instantaneous values of the 
populations of the states depend on time nearly periodically. The interesting effect of the periodicity of switchings between the states was nicely demonstrated by Zhou et al. [59] through measurements of the distribution of escape times in a periodically driven system (3), (17).

The above arguments provide an answer to the question formulated in [13] about the possibility of a "quasideterministic" periodic switchings, promoted by noise, in response to a weak periodic driving and establish the range of the parameters where such switchings occur.

Strong modulation of the populations gives rise to a strong modulation of the coordinate of the system. For a dynamically weak force the intrawell response is still described by linear response theory, and the ensemble-average value of the coordinate

$$
\langle q(t)\rangle=\sum_{n=1,2} w_{n}(t)\left(q_{n}+A \operatorname{Re} \chi_{n}(\Omega) e^{-i \Omega t}\right)
$$

where $q_{n}$ are the equilibrium positions in the states $n=1,2$ and $\chi_{n}(\Omega)$ are the intrawell susceptibilities. The coordinate $\langle q(t)\rangle$ of a fluctuating bistable system driven by a relatively weak sinusoidal force $A \cos \Omega t$ as obtained from (27) (dotted curve) is compared to the result of a digital simulation (full curve) in Fig.7 taken from Ref. [22]. It is obvious from Fig.7 that the time dependence of the coordinate is close to a square wave, as expected. A convenient numerical algorithm for investigating the redistribution of the system over the stable states induced by a comparatively weak force valid for arbitrary $\left|g_{1,2}\right|$ was considered in [22].

\section{B. Noise-enhanced heterodyning}

In heterodyning, two high-frequency fields, one of them a signal and the other a reference field, are mixed nonlinearly to generate a signal at their difference frequency. The frequencyselective response and the increase of the signal-to-noise ratio with increasing noise intensity in bistable systems make it interesting to apply the idea of stochastic resonance to heterodyning so as to obtain a new form of the phenomenon, noise-enhanced heterodyning (NEH), which will be also highly frequency selective. In other words, the idea behind NEH [12] is to mix the signal and the reference field via a "dynamical" nonlinearity of a bistable system, and then to single out the signal at the difference frequency and to enhance its signal-to-noise ratio via fluctuational transitions between coexisting stable states.

We shall illustrate the effect of NEH on a simple model of an overdamped fluctuating bistable system (3) driven by high-frequency forces that stand for the reference and input signals. In the presence of such forces the equation of motion (3) is modified to

$$
\dot{q}=-U^{\prime}(q)+A_{\text {ref }} q \cos \omega_{0} t+A_{\text {in }} \cos \left[\left(\Omega+\omega_{0}\right) t+\phi_{\text {in }}\right]+\xi(t)
$$

Here, the term $\propto A_{\text {ref }}$ is the reference signal of a given frequency $\omega_{0}$ (the corresponding force being applied multiplicatively), and that $\propto A_{\text {in }}$ is the high-frequency input signal (applied additively). In the general case the amplitude $A_{\text {in }}$ and the phase $\phi_{\text {in }}$ depend on time, but to characterize heterodyning one can assume them to be time-independent and investigate the heterodyne signal in the system at the difference frequency $\Omega$, with $\Omega \ll \omega_{0}$.

Simple analytical results can be obtained provided that the frequency $\omega_{0}$ is high compared to the reciprocal relaxation time of the system, $\omega_{0} \gg t_{\mathrm{rel}}^{-1}$. In this case the motion of the system consists of fast oscillations at frequency $\omega_{0}$ (and its overtones) superimposed on a slow motion. The equation for the slowly varying (on the time scale $\sim \omega_{0}^{-1}$ ) component 


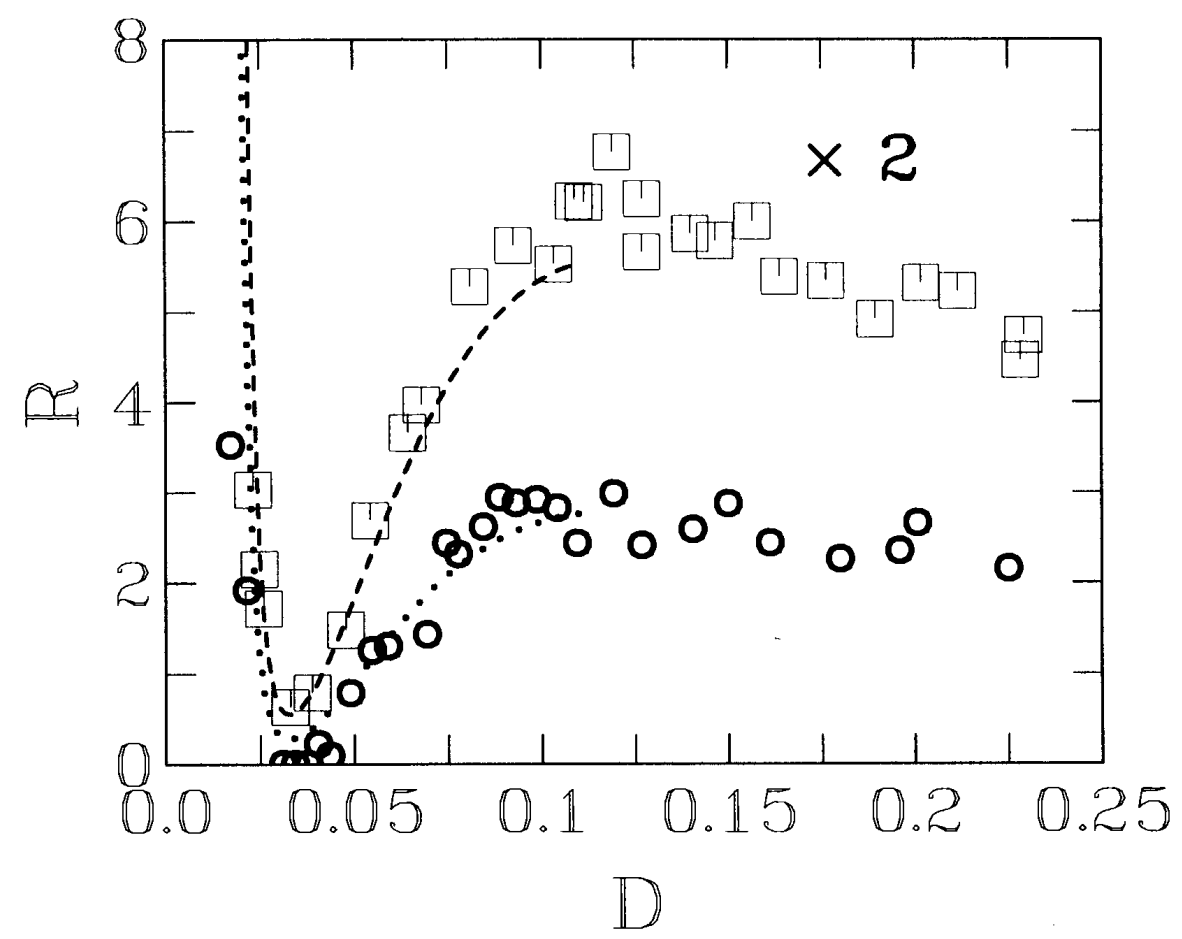

Figure 8: Noise-induced heterodyning for white (circles) and high-frequency (boxes) noise (after [12]). The dashed and dotted lines are theory. The potential simulated is the symmetrical double-well potential (17). The data refer to the reference frequency $\omega_{0}=1.885$. The heterodyne frequency $\Omega=0.008$.

of the coordinate $q^{(s m)}(t)$ can be obtained by the standard methods of nonlinear mechanics [60], which yield

$$
\dot{q}^{(s m)}=-U^{\prime}\left(q^{(s m)}\right)+A \cos \left(\Omega t+\bar{\phi}_{\text {in }}\right)+\bar{\xi}(t), \quad A=\frac{A_{\text {ref }}}{2 \omega_{0}} A_{\text {in }}, \quad \bar{\phi}_{\text {in }}=\phi_{\text {in }}-\frac{1}{2} \pi .
$$

We shall assume that the noise $\bar{\xi}(t)$ in (29) is Gaussian, with correlation time $t_{\text {cor }} \ll$ $\Omega^{-1},\left[W_{n m}^{(0)}\right]^{-1}$ where $W_{n m}^{(0)}$ is the probability of the fluctuational transition $n \rightarrow m$ for $A_{\text {in }}=$ 0 . In fact, the noise $\bar{\xi}(t)$ contains both the low-frequency and the rectified high-frequency components of the noise $\xi(t)$ in (28) (the latter components are shifted in frequency by $\omega_{0}$ and multiplied by $A_{\text {ref }} / 2 \omega_{0}$; in practice there is often high-frequency noise superimposed on the input signal, so that these components are important.

Eq. (29) is of precisely the same form as the equation of overdamped periodically driven motion in a double-well potential considered above. Therefore all the arguments of Secs.1,2 apply, and the signal-to-noise ratio for the signal in the system at the frequency $\Omega \ll$ $t_{\text {rel }}^{-1}$ should increase with the increasing noise intensity in a certain range of the latter. In particular, if the noise $\bar{\xi}(t)$ is white with intensity $D$, the dependence of the signal-to-noise ratio on $D$ for small enough amplitudes $A$ in the case of a symmetric double-well potential $U(q)$ should be given by Eq.(18).

The above arguments have been tested by means of analog electronic experiments [12]. In Fig. 8 measurements of the signal-to-noise ratio in heterodyning are presented for the two 
types of noise $\xi(t)$ : a broad-band (white) noise and a noise with a comparatively broad band centered at the frequency $\omega_{0}$. In both cases a strong enhancement of the heterodyne signal and, of the signal-to-noise ratio, induced by the noise is clearly seen, and is in good agreement with the theory.

\section{Conclusions}

It follows from the results discussed above that stochastic resonance may be understood and described in terms that are traditional for statistical physics, including linear response theory in particular, and that SR has its well-known counterparts in condensed-matter physics. In fact, of course, the possibility of increasing the response of a system to an external forcing by increasing the intensity of its fluctuations (temperature) is well known from daily experience: e.g., materials are often heated up prior to processing; light absorption in semiconductors and dielectrics increases with increasing temperature, in certain frequency ranges, and there are many other examples of the same kind. However, when thought in terms of signal processing, and particularly when one considers the noise-induced enhancement not only of a signal, but also of the signal-to-noise ratio, one can understand the interest SR has attracted.

The use of noise to control the response of a system is particularly effective when the dependence of the response on the noise intensity is exponentially sharp, as in the case of biand multistable systems where the noise intensity determines the probabilities of transitions between the states. It is in such systems that a sharp increase, not only in the signal (by orders of magnitude), but also in the signal-to-noise ratio (by more than an order of magnitude) has been achieved. This paves the way for the application of SR-displaying systems to signal processing, and also for revealing the role such systems may play in signal processing in biological systems.

One of the most interesting problems related to SR is the problem of large fluctuations, in particular the fluctuations that give rise to transitions between coexisting stable states. Although this is a traditional problem of condensed-matter physics (it includes nonadiabatic transitions, small-polaron transport, reorientation of defects and quantum diffusion, etc), many questions have not been resolved yet and are under active discussion (cf. Ref. [61]). Even more questions arise when this problem is considered for systems away from thermal equilibrium (cf. Ref. [62] and references therein). Here, many important problems are to be found that have not even been addressed to the best of our knowledge, e.g. the possibility of the onset of SR in systems driven by shot noise. We believe that the investigation of these problems and, more broadly, the analysis of the interplay of nonlinear dynamics and fluctuations will constitute an important direction in physics at least until early in the next century.

Acknowledgements

We are grateful to $\mathrm{R}$ Landauer who draw our attention to the earlier research on anelastic relaxation in solids, and to A Longtin and $\mathrm{K}$ Wiesenfeld for valuable comments on an earlier version of the manuscript. MID is grateful for support received from Stanford University; DGL would like to acknowledge the hospitality of Lancaster University. The research was supported in part by the Science and Engineering Research Council (UK), by the Royal Society of London, by the European Community DGXII, and by the Ministry of Education and Science of Russia. 


\section{References}

[1] Special issue of J. Stat. Phys. 70, nos. 1/2 (1993).

[2] A. Simon and A. Libchaber, Phys. Rev. Lett. 68, 3375 (1992).

[3] N.G. Stocks, P.V.E. McClintock, and S.M. Soskin, Europhys. Lett. 21, 395 (1993);N.G. Stocks, N.D. Stein, and P.V.E. McClintock, J. Phys. A 26, L385 (1993).

[4] A. Hibbs, E.W. Jacobs, J. Bekkedahl, A. Bulsara, and F. Moss, Proceedings of the AIP Conference on Noise in Physical Systems and $1 / f$ Fluctuations (AIP Press, New York, 1993), p.720; A.D. Hibbs, A.L.Singsaas, E.W. Jacobs, A.R. Bulsara, J.J. Bekkedahl and F. Moss, "Stochastic resonance in a superconducting loop with a Josephson junction", to be published.

[5] J.K. Douglass, L. Wilkens, E. Pantazelou, and F. Moss, Nature, 365, 337 (1993).

[6] T.L. Carroll and L.M. Pecora, Phys. Rev. Lett. 70, 576 (1993); Phys. Rev. E 47, 3941 (1993).

[7] M.I. Dykman, D.G. Luchinsky, P.V.E. McClintock, N.D. Stein, and N.G. Stocks, JETP Lett. 58, 145 (1993); M.I. Dykman, D.G. Luchinsky, R. Mannella, P.V.E. McClintock, N.D. Stein, and N.G. Stocks, Phys. Rev. E 49, 1198 (1994).

[8] R. Löfstedt and S.N. Coppersmith, Phys. Rev. Lett. 72, 1947 (1994).

[9] G.B. Alers, N.O. Birge, and B. Golding, submitted to Phys. Rev. Lett..

[10] K. Wiesenfeld, D. Pierson, E. Pantazelou, C. Dames, and F. Moss, Phys. Rev. Lett. 72, 2125 (1994).

[11] R.N. Mantegna and B. Spagnolo, Phys. Rev. 49, R1792 (1994).

[12] M.I. Dykman, G.P. Golubev, D.G. Luchinsky, P.V.E. McClintock, N.D. Stein, and N.G. Stocks, Phys. Rev. E 49, 1935 (1994).

[13] R.Benzi, A.Sutera, and A.Vulpiani, J. Phys. A 14, L453 (1981).

[14] C.Nicolis, Tellus 34, 1 (1982).

[15] R.Benzi, G.Parisi, A.Sutera, and A.Vulpiani, Tellus 34, 10 (1982).

[16] S. Fauve and F. Heslot, Phys. Lett. A 97, 5 (1983).

[17] B. McNamara, K. Wiesenfeld and R. Roy, Phys. Rev. Lett. 60, 2626 (1988).

[18] J.D. Hays, John Imbrie, and N.J. Shackleton, Science 194, 1121 (1976).

[19] M. Milankovitch, K. Serb. Akad. Beogr. Spec. Publ. 132 (1941).

[20] H. Kramers, Physica 7, 284 (1940).

[21] B. McNamara and K. Wiesenfeld, Phys. Rev. A 39, 4854 (1989). 
[22] M.I. Dykman, R. Mannella, P.V.E. McClintock, N.D. Stein, and N.G. Stocks, Phys. Rev. E 47, 1629 (1993).

[23] G.Debnath, T.Zhou, and F.Moss, Phys. Rev. A 39, 4323 (1989); L.Gammaitoni, F.Marchesoni, E.Menichella-Saetta, and S.Santucci, Phys. Rev. Lett. 62, 349 (1989); G.Vemuri and R.Roy, Phys Rev. A 39, 4668 (1989); C. Presilla, F. Marchesoni, and L. Gammaitoni, Phys. Rev. A 40, 2105 (1989).

[24] M.I.Dykman, R.Mannella, P.V.E.McClintock, and N.G.Stocks, Phys. Rev. Lett. 65, 2606 (1990); M.I.Dykman, P.V.E. McClintock, R.Mannella, and N.G.Stocks, JETP Lett. 52, 144 (1990)

[25] V.S. Anischenko, M.A. Safonova, and L.O. Chua, Int. J. Bifurcations Chaos 2, 397 (1992).

[26] M.I.Dykman, A.L.Velikovich, G.P.Golubev, D.G.Luchinsky, and S.V.Tsuprikov, JETP Lett. 53, 193 (1991).

[27] A. Fioretti, R. Mannella, L. Guidoni, L. Fronzoni, and E. Arimondo, J. Stat. Phys. 70, 799 (1993).

[28] E. Ippen, J. Linder, and W.L. Ditto, J. Stat. Phys. 70, 437 (1993).

[29] L. Gammaitoni, M. Martinelli, L. Pardi, and S. Santucci, Phys. Rev. Lett. 67, 1799 (1991).

[30] E.K. Sadykov, J. Phys.: Condens. Matter 4, 3295 (1992); Yu.L. Raikher and V.I. Stepanov, J. Phys.: Condens. Matter 6, 4137 (1994).

[31] S.T. Vohra and F. Bucholtz, J. Stat. Phys. 70, 413 (1993).

[32] A. Longtin, A. Bulsara, and F. Moss, Phys. Rev. Lett. 67, 656 (1991).

[33] F. Moss and A. Bulsara, Proceedings of the AIP Conference on Noise in Physical Systems and $1 / f$ Fluctuations (AIP Press, New York, 1993), p.703, and references therein.

[34] A. Longtin, J. Stat. Phys. 70, 309 (1993); D.R. Chialvo and A.V. Apkarian, J. Stat. Phys. 70, 375 (1993).

[35] A.R. Bulsara and G. Schmera, Phys. Rev. E 47, 3734 (1993).

[36] L.D. Landau and E.M. Lifshitz, Statistical Physics, 3rd ed., Part 1 (Pergamon, New York 1980).

[37] M.I. Dykman, D.G. Luchinsky, P.V.E. McClintock, N.D. Stein, and N.G. Stocks, Phys. Rev. A 46, R1713 (1992).

[38] A.R. Bulsara, S.B. Lowen and C.D. Rees, Phys. Rev. E 49, 4989 (1994).

[39] Z. Gingl, L.B. Kiss and F. Moss, "Non-dynamical stochastic resonance: theory and experiments with white and arbitrarily coloured noise", to be published. 
[40] M.I.Dykman and M.A.Krivoglaz, in Soviet Physics Reviews, ed I.M.Khalatnikov (Harwood, New York 1984) vol.5, p.265; M.I. Dykman and P.V.E. McClintock, Physica D 58, 10 (1992).

[41] S.M. Soskin, Physica A 180, 386 (1992); N.G. Stocks, N.D. Stein, S.M. Soskin, and P.V.E. McClintock, J. Phys. A 25, L1119 (1992).

[42] L.B. Kiss, Z. Gingl, Z. Márton, J. Kertész, F. Moss, G. Schmera, and A. Bulsara, J. Stat. Phys. 70, 451 (1993).

[43] P. Debye, Polar Molecules (Dover Publications, Inc.1929).

[44] A.S. Nowick and B.S. Berry, Anelastic Relaxation in Crystalline Solids (Academic Press, New York 1972).

[45] C. Wert and C. Zener, Phys. Rev. 76, 1169 (1949).

[46] J.L. Snoek, Physica 8, 711 (1941); Physica 9, 862 (1942).

[47] M.I. Dykman, R. Mannella, P.V.E. McClintock, and N.G. Stocks, Phys. Rev. Lett. 68, 2985 (1992).

[48] R. F. Fox and Y. Lu, Phys. Rev. E 48, 3390 (1993).

[49] H.M. Gibbs, Optical Bistability: Controlling Light with Light, Academic Press, New York, 1985.

[50] M.I. Dykman and M.A. Krivoglaz, Sov. Phys. JETP 50, 30 (1979).

[51] M.I. Dykman, Phys. Rev. A 42, 2020 (1990); M.I.Dykman and K.Lindenberg, in Contemporary Problems of Statistical Physics, edited by G. Weiss (SIAM, Philadelphia 1994), p.41.

[52] J.F. Luciani and A.D. Verga, Europhys. Lett. 4, 255 (1987); M.M. Klosek-Dygas, B.J. Matkowsky, and Z. Schuss, SIAM J. Appl. Math 48, 425 (1988); A.J. Bray and A.J. McKane, Phys. Rev. Lett. 62, 493 (1989); S. J. B. Einchcomb and A.J. McKane, Phys. Rev. E 49, 259 (1994); K. Lindenberg, B. J. West, and G. P. Tsironis, Rev. Solid State Sci. 3, 143 (1989).

[53] N.G. van Kampen, Stochastic Processes in Physics and Chemistry (Elsevier, Amsterdam, 1990); H. Risken, The Fokker-Planck Equation 2nd edition (Springer, Berlin, 1989).

[54] (a) G. Hu, H. Haken, and C.Z. Ning, Phys. Lett. A 17221 (1992); (b) M.I. Dykman, H. Haken, Hu Gang, D.G. Luchinsky, P.V.E. McClintock, C.Z. Ning, N.D. Stein, and N.G. Stocks, Phys. Lett. A, 180, 332 (1993).

[55] P. Hänggi and H. Thomas, Phys. Rep. 88, 207 (1982).

[56] M. Morillo and J. Gòmez-Ordòñez, Phys. Rev. Lett. 71, 9 (1993).

[57] P. Jung and P. Hänggi, Europhys. Lett. 8, 505 (1989). 
[58] A. Neiman and L. Schimansky-Geier, Phys. Rev. Lett. 72, 2988 (1994).

[59] T. Zhou, F. Moss, and P. Jung, Phys. Rev. A 42, 3161 (1990).

[60] L.D. Landau and E.M. Lifshitz, Mechanics (Pergamon, London 1976).

[61] Quantum tunnelling in Condensed Media, ed. by Yu. Kagan and A.J. Leggett (Elsevier, Amsterdam, 1992).

[62] R.S. Maier and D.L. Stein, Phys. Rev. Lett. 69, 3691 (1992); Phys. Rev. E 48, 931 (1993); V. A. Chinarov, M. I. Dykman and V. N. Smelyanskiy, Phys. Rev. E 47, 2448 (1993); R.S. Maier and D.L. Stein, Phys. Rev. Lett. 71, 1783 (1993); M.I. Dykman, M. Millonas, and V.N. Smelyanskiy, Phys. Lett. A (1994). 